\section{Os custos da violência para o sistema público de saúde no Brasil: informações disponíveis e possibilidades de estimação}

\author{
The cost of violence for the public health system in \\ Brazil: available data and possibilities for \\ estimation
}

1 Instituto de Pesquisa Econômica Aplicada, Rio de Janeiro, Brasil. 2 Instituto Brasileiro de Geografia e Estatística Rio de Janeiro, Brasil.

Correspondência R. I. Rodrigues Instituto de Pesquisa Econômica Aplicada. Av. Presidente Antônio Carlos

51, 10o andar,

Rio de Janeiro, $R J$ 20020-010, Brasil.

rute.imanishi@ipea.gov.br

\begin{abstract}
The Brazilian public budget for health was $R \$$ 53 billion (approximately U\$20 billion) in 2003. What share of this total was to treat victims of violence? It is impossible to estimate that proportion directly, due to limitations in the available data. This paper proposes a methodology to estimate the cost of violence for the public health system, using data from the Unified National Health System, the government budget, and the demand for public medical services (National Household Sample Survey). According to our estimates, in 2004 the costs of treatment for victims of external causes, aggressions, and traffic accidents were $R \$$ 2.2 billion, $R \$ 119$ million, and $R \$ 453$ million, respectively. These findings were approximately four times higher than those observed in other studies.
\end{abstract}

Single Health System; Information Systems; Violence
Rute Imanishi Rodrigues 1

Daniel Ricardo de Castro Cerqueira 1

Waldir Jesus de Araújo Lobão 2

Alexandre Xavier Ywata de Carvalho 1

\section{Introdução}

O orçamento público em saúde era da ordem de 53 bilhões de Reais em 2003, considerando as três esferas de governo, conforme apontado na Tabela 1. Naquele ano, os valores da produção ambulatorial e das internações hospitalares, financiadas pelo Sistema Único de Saúde (SUS), representaram $13 \%$ e $11 \%$ do total de gastos públicos com saúde, respectivamente. Na Tabela 1, podemos observar que os valores referentes às internações por causas externas representaram cerca de $1 \%$ do total de gastos governamentais com a saúde. Esses números correspondem à remuneração pelos serviços produzidos por unidades de saúde vinculadas ao SUS (que seguem uma tabela padronizada de valores) e não incorporam os gastos correntes e investimentos referentes à manutenção das unidades, aquisição de equipamentos etc.

Duas limitações nos dados disponíveis dificultam o cálculo direto do custo da violência para o sistema de saúde pública no Brasil. Em primeiro lugar, as informações disponíveis não permitem uma distinção dos procedimentos - e custos associados - adotados para o tratamento das vítimas de violência daqueles atendimentos devido às demais causas de morbidade, no nível ambulatorial do SUS. De fato, o Sistema de Informações Ambulatoriais (SIA) incorpora desde consultas de atendimento básico até os procedimentos realizados por prontos-socorros e 
Tabela 1

Gastos públicos (em milhões de Reais) com a saúde. Brasil, 2000-2003.

\begin{tabular}{|c|c|c|c|c|c|c|c|c|c|c|}
\hline \multirow[t]{4}{*}{ Ano } & \multicolumn{8}{|c|}{ Remuneração por serviços produzidos * } & \multirow{3}{*}{\multicolumn{2}{|c|}{$\begin{array}{c}\text { Total de gastos públicos } \\
\text { (União, estados e } \\
\text { municípios) } \star \star\end{array}$}} \\
\hline & \multicolumn{4}{|c|}{ Internações (SIH) } & \multirow{2}{*}{\multicolumn{2}{|c|}{$\begin{array}{c}\text { Ambulatorial (SIA) } \\
\text { Total }\end{array}$}} & \multirow{2}{*}{\multicolumn{2}{|c|}{$\begin{array}{l}\mathrm{SIH}+\mathrm{SIA} \\
\text { Total }\end{array}$}} & & \\
\hline & \multicolumn{2}{|c|}{ Causas externas } & \multicolumn{2}{|c|}{ Total } & & & & & & \\
\hline & Reais & $\%$ & Reais & $\%$ & Reais & $\%$ & Reais & $\%$ & Reais & $\%$ \\
\hline 2000 & 352 & 1 & 4.887 & 14 & 5.698 & 17 & 10.585 & 31 & 34.069 & 100 \\
\hline 2001 & 362 & 1 & 5.096 & 13 & 4.992 & 12 & 10.088 & 25 & 40.013 & 100 \\
\hline 2002 & 407 & 1 & 5.406 & 12 & 5.714 & 12 & 11.120 & 24 & 46.805 & 100 \\
\hline 2003 & 472 & 1 & 5.862 & 11 & 6.736 & 13 & 12.598 & 23 & 53.624 & 100 \\
\hline
\end{tabular}

$\mathrm{SIH}$ : Sistema de Informações Hospitalares; SIA: Sistema de Informações Ambulatoriais.

* Fonte: Departamento de Informática do SUS (DATASUS);

** Fonte: Sistema de Informações sobre Orçamentos Públicos em Saúde (SIOPS).

ambulatórios de urgência/emergência, mas não classifica as informações de acordo com o tipo de doença (causa) do atendimento. Especialistas em saúde, como Jorge \& Koizumi ${ }^{1}$, avaliam que o atendimento de urgência/emergência represente parte importante dos gastos com as vítimas de acidentes e violências.

Em segundo lugar, os pagamentos por serviços produzidos, que são registrados no SIA e no Sistema de Informações Hospitalares (SIH), representam apenas uma parte das despesas governamentais destinadas à rede de atendimento pública. Uma parcela importante dos recursos provém dos orçamentos públicos estaduais e municipais que complementam os recursos repassados para a "remuneração por serviços produzidos" a partir do Fundo Nacional de Saúde (FNS).

Ou seja, os recursos registrados nos sistemas SIA e SIH referem-se, sobretudo, aos repasses do Ministério da Saúde - por intermédio do FNS - para estados, municípios e prestadores diretos dos serviços (rede contratada). Além disso, pode haver repasses do governo federal destinados a programas de prevenção e/ou atendimento a vítimas de determinadas causas que não são contabilizados nos sistemas SIA e SIH (uma vez que independem dos registros de produção hospitalar e ambulatorial) ${ }^{2}$.

O objetivo deste trabalho é propor um método para estimar os custos do atendimento curativo às vítimas de acidentes e violências (ou seja, exclusive os gastos com prevenção), que utilize as bases de dados disponíveis sobre os gastos públicos com a saúde. Para tanto, propomos uma determinada medida da demanda por atendimento ambulatorial devido a causas externas utilizando dados do suplemento de saúde da Pesquisa Nacional por Amostra de Domicílios (PNAD), e uma forma de contabilizar os "recursos próprios" destinados aos atendimentos hospitalar e ambulatorial por estados e municípios (parcela não contabilizada nos recursos registrados no SIA e SIH) utilizando dados do Sistema de Informações sobre Orçamentos Públicos em Saúde (SIOPS).

\section{O estado da pesquisa sobre o tema}

$\mathrm{O}$ atendimento às vítimas de acidentes e violências pode se dar no nível da "prevenção", na fase "pré-hospitalar (pré-internação)", "hospitalar (internação)” e "pós-hospitalar (pós-internação)". Quanto maior o grau de mortalidade das vítimas de causas externas antes de qualquer atendimento médico, maior a desproporção en tre os dados de mortalidade e os de morbidade por tais causas e, conseqüentemente, menor o impacto sobre o sistema curativo de saúde e seus custos. Com efeito, sobretudo nos casos de violência intencional (como agressões), a alta letalidade faz com que a proporção dos casos seja mais importante sobre a mortalidade do que sobre o número de internações hospitalares. Como mostraram Gawryszewski et al. 3, em 2000 as agressões representaram $38 \%$ do total de mortos por causas externas e apenas $5,4 \%$ dos internados por tais causas (no âmbito do SUS). Também no caso dos acidentes de trânsito havia desproporção entre mortalidade e morbidade, porém de menor grau: $25 \%$ das mortes e $18,2 \%$ dos internados. O inverso ocorria com as quedas que correspondiam a $3,6 \%$ das mortes e $42,8 \%$ dos internados por causas externas.

Embora apenas uma parcela dos casos de violência intencional chegue a ser atendida pelos serviços de saúde, nas grandes cidades esta demanda é suficiente para "congestionar" os 
serviços de urgência e emergência - como relata Pinheiro ${ }^{4}$ - sem que isto se manifeste em um número muito alto de internações. Há ainda que considerar os casos de morte que ocorrem nos prontos-socorros e emergências dos hospitais, ou seja, no nível ambulatorial do atendimento.

Devido a essas dificuldades, a estimação dos custos dos acidentes e violências para o sistema de saúde tende a conter um componente desconhecido não desprezível, sobretudo no que diz respeito aos custos das causas externas nos atendimentos de urgência e emergência. Com efeito, esses custos estão agregados no gasto total com serviços ambulatoriais, para os quais não há informação direta sobre o "motivo do atendimento", mas é exatamente neste tipo de atendimento que pode ocorrer um grande número de casos que não são passíveis de verificação por meio das pesquisas sobre a demanda por serviços de saúde (como o suplemento de saúde da PNAD), já que é desconhecida a demanda dos pacientes que vieram a falecer.

Os pesquisadores que investigaram os custos das causas externas e/ou acidentes e violências sobre o sistema de saúde no Brasil adotaram metodologias diversas. Alguns autores analisaram os dados disponíveis sobre as internações hospitalares para esse grupo de causas, tais como Jorge \& Koizumi 1, Feijó \& Portela 5, Mendonça et al. 6 e Iunes 7. Esse tipo de abordagem tem a vantagem de trabalhar com informações bastante detalhadas sobre todas as internações realizadas pelo SUS, que são registradas nas guias de Autorização para Internação Hospitalar (AIH) do SIH. Com base nesses dados, que são disponibilizados pelo Departamento de Informática do SUS (DATASUS), é possível recuperar os procedimentos realizados em cada internação e seus respectivos valores, além do motivo de saída (se alta ou óbito), local de internação, características dos pacientes, sendo todas as informações desagregadas por tipo de causa de acordo com a Classificação Internacional de Doenças (CID). Há, porém, duas lacunas importantes nesse tipo de abordagem dos custos. Em primeiro lugar, nem todas as despesas necessárias para custear as internações hospitalares passam pelos registros das AIHs. Com efeito, nas AIHs os valores correspondentes aos custos das internações são baseados em uma "tabela de procedimentos" do SUS, e funcionam como a principal referência para os repasses dos recursos federais destinados ao custeio das internações hospitalares aos estados, municípios ou diretamente aos próprios prestadores de serviços (hospitais). Porém, o custeio do sistema hospitalar muitas vezes conta com despesas realizadas por estados e municípios com recursos próprios, seja para pagamento de pessoal, seja para outras despesas correntes dos hospitais. Em segundo lugar, os registros das internações nada informam sobre o atendimento ambulatorial às vítimas, seja em postos de saúde, seja em ambulatórios de urgência e emergência de hospitais.

Seguindo outra linha de pesquisa, alguns autores se propuseram a medir os custos do atendimento de urgência e emergência de vítimas de causas externas por meio de estudos de caso, como Deslandes et al. 8, Martins \& Andrade 9. Nesses trabalhos foram selecionados determinados hospitais cujos serviços de urgência e emergência eram importantes no respectivo município e foram avaliados os atendimentos às vítimas de causas externas por meio dos prontuários médicos, nos quais foram coletadas informações in loco sobre todos os procedimentos médicos, medicamentos, além de informações sobre alimentação, horas de trabalho dos médicos e outras, assim como seus respectivos custos. A vantagem desse método é a de trazer informações sobre o atendimento de urgência e emergência às vítimas de causas externas, que é apontado como uma das faces mais problemáticas da questão do atendimento a este tipo de causa. Porém, os resultados dos estudos de caso dificilmente podem ser generalizados, pois em geral os hospitais têm características de demanda e de custos bastante específicas devido às suas especialidades.

Finalmente, Carneiro \& Phebo ${ }^{10}$ propuseram uma metodologia que utiliza ambos os métodos já citados. Com o propósito de estimar os custos do atendimento às vítimas de violência intencional no Município do Rio de Janeiro, os autores contabilizaram os valores referentes às internações do SUS e também coletaram informações sobre o atendimento de urgência e emergência de um conjunto de hospitais de referência para causas externas, agregando ainda estimativas da PNAD sobre a demanda por serviços ambulatoriais para estimar o número de atendimentos pela rede ambulatorial. Além disso, supuseram um "ajuste para cima" da tabela do SUS para os custos dos procedimentos ambulatoriais e das AIHs como forma de contabilizar os gastos provenientes do tesouro estadual ou municipal para pagamento de pessoal das redes hospitalar e ambulatorial. Assim, o trabalho buscou incorporar tanto as internações quanto o atendimento ambulatorial, e ainda as despesas não registradas nos sistemas de informações do DATASUS, ou seja, os recursos próprios despendidos por governos municipais e estaduais. Esses autores basearamse em parâmetros arbitrários para os ajustes necessários, tanto para o número de atendimentos ambulatoriais quanto para o acréscimo de valor não contabilizado nos registros de pagamentos 
por serviços produzidos. Para estimar o número dos atendimentos ambulatoriais, o trabalho supõe que cada vítima tratada ambulatorialmente tenha recebido em média três procedimentos ou atendimentos. Para contabilizar os recursos estaduais e municipais não contabilizados no DATASUS, os autores propõem que estes correspondem aos gastos com pessoal que seriam cerca de $60 \%$ dos gastos hospitalares e $80 \%$ dos gastos em unidades ambulatoriais.

\section{Metodologia}

Em nosso exercício supomos algumas identidades contábeis entre as informações dos orçamentos públicos em saúde e os valores registrados nos sistemas do DATASUS como ponto de partida para estimar as despesas públicas com atendimento às vítimas de causas externas.

Em primeiro lugar, supomos uma relação de identidade entre, de um lado, as despesas governamentais agregadas na subfunção da saúde "serviços hospitalares e ambulatoriais" e registradas nos orçamentos da saúde e, de outro, os valores correspondentes aos "custos" destes serviços. Tais "custos" referem-se tanto à produção hospitalar e à produção ambulatorial, registrados respectivamente nos sistemas SIH e SIA, quanto a um componente relativo aos "custos do sistema”.

A lógica dessa identidade é simples: os gastos governamentais registrados nos orçamentos como "serviços hospitalares e ambulatoriais" deveriam representar a totalidade dos gastos públicos com estes serviços, enquanto os valores registrados nos sistemas SIA e SIH representam apenas a parcela referente à "remuneração por serviços produzidos”. Assim, o resíduo da identidade deveria significar o componente do gasto governamental necessário para manter os ambulatórios e hospitais, porém, não contabilizados no DATASUS.

Para simplificar a notação utilizamos os termos "custos das internações hospitalares" - C(I) - e "custos dos atendimentos ambulatoriais" $\mathrm{C}(\mathrm{A})$ - para representar os valores registrados nos sistemas SIH e SIA, respectivamente. É importante ressaltar que em nosso modelo os custos dos atendimentos hospitalares e ambulatoriais excedem os valores registrados no DATASUS na medida do componente "custos do sistema" - C(S).

A identidade entre custos e despesas é expressa por meio das equações 1, 2 e 3 .

A equação 1 descreve a "Despesa Total do Sistema Hospitalar e Ambulatorial” (DT) do SUS como o somatório destas despesas para os três níveis de governo, como se segue:
(1) $\mathrm{DT}=\mathrm{D}_{\text {federal }}+\mathrm{D}_{\text {estadual }}+\mathrm{D}_{\text {municipal }}-\mathrm{E}$ em que: $D_{\text {federal }}$ representa as despesas do governo federal, $D_{\text {estadual }}$ as despesas dos governos estaduais e $D_{\text {municipal }}$ as dos governos municipais e $E$ representa o erro que pode ocorrer devido à dupla contagem de recursos transferidos entre as esferas governamentais.

A equação 2 descreve os "Custos do Atendimento Hospitalar e Ambulatorial” (CT) como a soma dos custos com o atendimento ambulatorial, das internações hospitalares e a variável "custos do sistema", como se segue.

(2) $\mathrm{CT}=\mathrm{C}(\mathrm{A})+\mathrm{C}(\mathrm{I})+\mathrm{C}(\mathrm{S})$

em que: C(A) representa o valor total da produção ambulatorial dado pelo SIA, que equivale ao custo médio dos procedimentos ambulatoriais vezes o número de procedimentos: $\mathrm{C}(\mathrm{A})=\mathrm{c}(\mathrm{a}) \mathrm{q}(\mathrm{a}) ; \mathrm{C}(\mathrm{I})$ representa $o$ valor total das internações hospitalares dado pelo SIH, que equivale ao custo médio das internações vezes o número de internações: $\mathrm{C}(\mathrm{I})=\mathrm{c}(\mathrm{i}) \mathrm{q}(\mathrm{i})$.

O componente $\mathrm{C}(\mathrm{S})$ representa o resíduo contábil da identidade DT $=$ CT. Dado que os pagamentos relativos a C(A) e C(I) são efetuados com recursos transferidos do orçamento federal, então $\mathrm{C}(\mathrm{S})$ pode ser interpretado como o componente relativo aos "recursos próprios" despendidos por estados e municípios que complementam o custeio dos serviços ambulatoriais e hospitalares.

(3) $\mathrm{DT}=\mathrm{C}(\mathrm{A})+\mathrm{C}(\mathrm{I})+\mathrm{C}(\mathrm{S})$

A partir da equação 3 derivamos o "coeficiente de gastos" (M), dividindo ambos os lados da equação por $[\mathrm{C}(\mathrm{A})+\mathrm{C}(\mathrm{I})]$ :

$\mathrm{DT}=[\mathrm{C}(\mathrm{A})+\mathrm{C}(\mathrm{I})] \mathrm{M}$

em que:

$$
\mathrm{M}=1+\frac{\mathrm{C}(\mathrm{S})}{\mathrm{C}(\mathrm{A})+\mathrm{C}(\mathrm{I})}
$$

$O$ coeficiente de gastos significa que para cada 1 Real gasto em C(A) ou C(I) um valor extra é despendido para o pagamento dos C(S). Em outras palavras, o coeficiente de gastos faz um ajuste para cima dos custos hospitalares e ambulatoriais sempre que C(S) for maior que zero. Vale lembrar que C(S) é a diferença entre as despesas registradas nos orçamentos da saúde como serviços hospitalares e ambulatoriais e o total dos gastos registrados no SIA e no SIH.

Finalmente, como nos interessa aferir as despesas com determinadas causas, representamos as despesas totais como o somatório das despesas com cada causa específica $j$, como expresso na equação 4 .

(4) $D T=\sum_{j}^{n} d_{j}=\sum_{j}^{n}\left[c_{j}(a) q_{j}(a)+c_{j}(i) q_{j}(i)\right] M$

Similarmente, podemos representar os custos de cada causa específica $j$ como o resultado 
dos pagamentos por serviços produzidos nos sistemas hospitalar e ambulatorial para atender a causa $j$ vezes o coeficiente de gastos:

(5) $\mathrm{C}_{\mathrm{j}}=\left[\mathrm{c}_{\mathrm{j}}(\mathrm{a}) \mathrm{q}_{\mathrm{j}}(\mathrm{a})+\mathrm{c}_{\mathrm{j}}(\mathrm{i}) \mathrm{q}_{\mathrm{j}}(\mathrm{i})\right] \mathrm{M}$

A equação 5 evidencia que o único componente que permanece desconhecido é a parcela dos gastos ambulatoriais devida a cada doença (ou causa), $c_{j}(a) q_{j}(a)$, uma vez que $M$ deriva dos orçamentos públicos em saúde e das informações totalizadas para a produção hospitalar e ambulatorial; e os gastos hospitalares por doença/causa, $c_{j}(i) q_{j}(i)$, podem ser observados diretamente por meio das informações do SIH.

O componente do custo ambulatorial por tipo de causa pode ser estimado com base na seguinte equação:

(6) $c_{j}(a) q_{j}(a)=C(A) \alpha_{j}(a) \beta_{j}(a)$

em que: $\alpha_{j}$ representa a proporção da doença $j$ no número total de atendimentos do sistema ambulatorial:

$\alpha_{j}(a)=\frac{q_{j}(a)}{\sum q_{j}(a)}$

$\beta_{\mathrm{j}}$ representa o quociente entre o custo de tratamento da doença j e o custo médio de tratamento de todas as doenças:

$$
\beta_{j}(a)=\frac{c_{j}(a)}{-}
$$

Assim, as estimativas dos custos do atendimento aos doentes da causa $j$ dependem fundamentalmente dos valores atribuídos a $\alpha_{j}(a)$ e $\beta_{\mathrm{j}}$ (a). Na seção seguinte, estimamos os parâmetros $\alpha_{\mathrm{j}}$ (a) e $\beta_{\mathrm{j}}$ (a) e, subseqüentemente, os custos dos atendimentos às vítimas de causas externas.

\section{Dados utilizados}

Neste exercício, partimos dos dados relativos às despesas governamentais com saúde nas três esferas de governo utilizando os resultados fiscais da União, de todos os estados e para um conjunto dos municípios com as 100 maiores despesas na função saúde no ano de 2004. Tais dados são oriundos do Demonstrativo da Receita de Impostos e das Despesas Próprias com Saúde (documento do Relatório Resumido da Execução Orçamentária), disponibilizados pelo DATASUS por meio do SIOPS.

Tomamos as despesas na subfunção "Assistência Hospitalar e Ambulatorial" como representativas do total de despesas governamentais realizadas para as internações hospitalares, assim como para o atendimento da demanda por serviços ambulatoriais do SUS de média e alta complexidades. Entre tais despesas encontram- se as de pessoal, manutenção dos hospitais, equipamentos, medicamentos, materiais e etc, assim como investimentos e outros tipos de gastos destinados a estes sistemas. Não incluímos as despesas relativas à subfunção "atenção básica à saúde”, pois esta abrange principalmente procedimentos relativos à "prevenção de doenças" e não ao tratamento das mesmas 11.

É importante observar que as transferências intergovernamentais pouco afetam o somatório das despesas registradas na rubrica "assistência hospitalar e ambulatorial" do ponto de vista da dupla contagem. Isso porque a maior parte das transferências tem origem no governo federal, que repassa diretamente aos estados e municípios, mas não registra tais transferências como "Assistência Hospitalar e Ambulatorial". Com efeito, em 2004 o Ministério da Saúde transferiu cerca de 13,7 bilhões aos estados e municípios da gestão plena para despesas com Atendimento Hospitalar e Ambulatorial, porém, no demonstrativo daquele ano apenas 1,3 bilhão foi registrado como despesas desta rubrica pelo governo federal. Já as transferências dos estados para os municípios são de pequena monta e não alteram a distribuição das despesas de forma importante.

Cabe ressalvar que as informações coletadas pelo SIOPS têm caráter declaratório (referem-se às informações declaradas por estados e municípios) e, portanto, estão sujeitas a erros ou problemas de classificação ${ }^{12}$. Não obstante, os dados do SIOPS têm se mostrado importantes para os pesquisadores da área da saúde, sobretudo por serem a principal fonte de informações organizadas sobre os gastos de saúde dos governos estaduais e municipais. 13 .

O conjunto de 100 municípios incluídos no exercício totalizou gastos com saúde equivalentes a $50 \%$ do total de gastos municipais. Supusemos, então, que as despesas com assistência hospitalar e ambulatorial desse conjunto representam $50 \%$ do total de despesas municipais com esta subfunção (ou seja, o total das despesas de todos os municípios corresponderia a 2 vezes o valor gasto pelos 100 municípios selecionados). Para o conjunto de 100 municípios calculamos o total das transferências dos estados aos respectivos municípios como o componente de “erro" E.

Utilizamos também as informações disponíveis no SIA e no SIH que se referem ao valor total dos pagamentos por serviços produzidos por um e outro sistema, ou seja, a remuneração da produção ambulatorial e a referente às internações hospitalares.

O segundo passo desta estimativa foi definir um parâmetro para a proporção dos atendimentos relativos às causas externas para o conjunto 
dos atendimentos ambulatoriais do SUS, ou seja, $\alpha_{j}$ (a). Para tanto, usamos os dados do suplemento sobre saúde da PNAD (2003), que possibilita a estimação da demanda por serviços de saúde pela população brasileira. A PNAD discrimina apenas alguns "motivos" da demanda, entre elas "Acidente ou Lesão", ou seja, causas externas. Para os tipos de causas externas, há alguma informação para as "agressões", para os "acidentes de trabalho" e para os "acidentes de trânsito", porém estas apenas são registradas como "motivo da doença" para “doentes graves", ou seja, aqueles que declaram haver interrompido suas atividades normais no período de referência da pesquisa. Embora dependa da declaração da pessoa pesquisada, a PNAD apurou a incidência de atendimentos financiados pelo SUS e este foi o dado utilizado no exercício. Consideramos também que um atendimento que gerou internação significou um atendimento ambulatorial, ou seja, os internados contaram como parte da demanda ambulatorial. Cabe observar que entre os motivos principais da procura discriminados na PNAD aparece o grupo "Vacinação e Outros Atendimentos de Prevenção", porém, assim como retiramos as despesas com atenção básica à saúde do total de despesas com o sistema ambulatorial e hospitalar, também excluímos os atendimentos preventivos do cálculo da demanda total por serviços ambulatoriais.

Finalmente, para os custos dos atendimentos ambulatoriais relativos às causas externas utilizamos as informações das internações hospitalares do SUS (SIH). A razão entre o custo médio de internação de uma determinada causa e o custo médio do total de internações foi interpretado como o parâmetro $\beta_{\mathrm{j}}(\mathrm{a})$. Em outras palavras, supusemos que as diferenças nos custos de tratamentos das internações por diversas causas valeriam inclusive para o atendimento em nível ambulatorial.

Os dados utilizados estão discriminados na Tabela 2.

\section{Resultados}

Com base nos parâmetros estimados, os custos do tratamento com as vítimas de violência podem ser obtidos por meio da equação (5). Os resultados encontrados são apresentados na Tabela 3.

Para ter uma idéia da dimensão dessas estimativas cabe observar que no DATASUS o valor das internações hospitalares registradas como "causas externas", como "agressões" e como “acidentes de trânsito” em 2004 totalizaram 540

\begin{tabular}{|c|c|c|c|}
\hline Variável & Descrição & Valor & Fonte \\
\hline$D_{\text {federal }}$ & Despesa na subfunção "Assistência Hospitalar e Ambulatorial" & $\mathrm{R} \$ 1.372 .175 .000$ & SIOPS \\
\hline$D_{\text {estadual }}$ & Despesa na subfunção "Assistência Hospitalar e Ambulatorial" & $\mathrm{R} \$ 11.244 .537 .600$ & SIOPS \\
\hline $\mathrm{D}_{\text {municipal * }}$ & Despesa na subfunção "Assistência Hospitalar e Ambulatorial" & $\mathrm{R} \$ 7.614 .816 .900$ & SIOPS \\
\hline $\mathrm{E}$ & Transferências dos estados para os municípios * & $\mathrm{R} \$ 111.545 .000$ & SIOPS \\
\hline$C(A)$ & Valor total da produção ambulatorial & $\mathrm{R} \$ 6.581 .213 .300$ & SIA \\
\hline$C(I)$ & Valor total das internações hospitalares & $\mathrm{R} \$ 7.867 .733 .200$ & $\mathrm{SIH}$ \\
\hline$c_{j}(i) q_{j}(i)$, para $j=$ causas externas & Valor das internações hospitalares por causas externas & $\mathrm{R} \$ 540.197 .100$ & $\mathrm{SIH}$ \\
\hline$c_{j}(i) q_{j}(i)$, para $j=$ agressões & Valor das internações hospitalares por agressões & $\mathrm{R} \$ 37.857 .400$ & $\mathrm{SIH}$ \\
\hline$c_{j}(i) q_{j}(i)$, para $j=$ acidentes de trânsito & Valor das internações hospitalares por acidentes de trânsito & $\mathrm{R} \$ 106.524 .100$ & $\mathrm{SIH}$ \\
\hline$\alpha_{j}$, para $j=$ causas externas ** & Parcela da demanda por serviços de saúde, motivo "Acidentes e Lesões" & 0,0738 & PNAD \\
\hline$\alpha_{j}$, para $j=$ agressões $* \star \star$ & Parcela da demanda por serviços de saúde, motivo "Agressões" & 0,0024 & PNAD \\
\hline 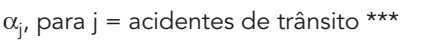 & Parcela da demanda por serviços de saúde, motivo "Acidentes de Trânsito" & 0,0123 & PNAD \\
\hline$\beta_{\mathrm{j}}$, para $\mathrm{j}=$ causas externas & Valor médio das AlHs por causas externas/valor médio das AlHs & 1,3 & $\mathrm{SIH}$ \\
\hline$\beta_{\mathrm{j}}$, para $\mathrm{j}=$ agressões & Valor médio das AlHs por agressões/valor médio das AlHs & 1,6 & $\mathrm{SIH}$ \\
\hline$\beta_{\mathrm{j}}$ para $\mathrm{j}=$ acidentes de trânsito & Valor médio das AlHs por acidentes de trânsito/Valor médio das AlHs & 1,6 & $\mathrm{SIH}$ \\
\hline
\end{tabular}

SIOPS: Sistema de Informações sobre Orçamentos Públicos em Saúde; SIA: Sistema de Informações Ambulatoriais; SIH: Sistema de Informações Hospitalares; PNAD: Pesquisa Nacional por Amostra de Domicílios; AlH: Autorização para Internação Hospitalar.

* Dados para o total dos municípios estimados a partir dos valores registrados para 100 municípios selecionados;

** Exclui demanda por prevenção;

*** Apenas pessoas que deixaram de realizar atividades de rotina nas semanas de referência. 
Tabela 3

Gastos públicos estimados de acordo com o tipo de causa.

\begin{tabular}{lc}
\hline Causa & Custo estimado \\
\hline Causas externas & $\mathrm{R} \$ 2.212 .224 .218$ \\
Agressões & $\mathrm{R} \$ 119.227 .409$ \\
Acidentes de trânsito & $\mathrm{R} \$ 453.228 .958$ \\
\hline
\end{tabular}

milhões, 37,8 milhões e 106,5 milhões de Reais, respectivamente. Ou seja, o custo estimado pelo exercício proposto foi 4,1 vezes maior que o custo das internações por causas externas, 3,2 vezes o custo das internações por agressões e 4,3 vezes maior que o custo das internações por acidentes de trânsito.

Nesse ponto é importante ressalvar que as estimativas para os atendimentos por "agressões" e "acidentes de trânsito" oriundas da PNAD são menos abrangentes que a dos atendimentos para "causas externas" em geral, uma vez que as primeiras referem-se apenas às pessoas que declararam ter deixado de realizar suas atividades normais no período de referência da pesquisa, o que corresponde a um terço do total que declara ter sido atendido em algum serviço de saúde. Além disso, pode ser considerável, sobretudo no caso das agressões, o número de pacientes dos serviços de urgência e emergência que vieram a falecer e que, obviamente, não são contados nas estimativas de demanda por serviços de saúde da PNAD.

A insuficiência de informações nos registros ambulatoriais, entretanto, nos impediu de realizar uma estimativa mais rigorosa dos atendimentos por tipo de causa na rede ambulatorial e, conseqüentemente, de seus custos. Assim como outros autores, utilizamos parâmetros externos ao SIH para realizar essa estimativa.

No que diz respeito à parcela dos gastos da saúde oriunda dos governos estaduais e municipais, a utilização dos dados do SIOPS (em que pesem suas imperfeições) nos permitiu alcançar um menor grau de arbitrariedade em relação a estudos anteriores, realizados quando estes dados ainda não estavam disponíveis. Encontramos um coeficiente de gastos de 1.9 , ou seja, os gastos totais com serviços ambulatoriais e hospitalares seriam cerca de 2 vezes maiores que os registrados nos sistemas SIA e SIH.

\section{Considerações finais}

Os registros de pagamentos das internações hospitalares por causas externas representaram cerca de $1 \%$ do total de gastos com a saúde pública no Brasil no período 1998-2004. Os valores referentes ao pagamento das AIHs, entretanto, representam apenas uma parcela do total de gastos do sistema público de saúde destinado ao atendimento das vítimas de violência. Uma parcela importante dos gastos refere-se ao atendimento ambulatorial, inclusive de urgência e emergência, para o qual não existem - no DATASUS - informações sobre gastos organizadas por tipo de causa/doença. Além disso, os registros de pagamentos das AIHs não contemplam os gastos efetuados por estados e municípios com o SUS.

Propusemos uma metodologia para estimar os custos totais do atendimento às vítimas de violência pelo sistema público de saúde, com vistas a contornar tais dificuldades e ampliar nosso conhecimento da amplitude de tais custos. Tal metodologia utilizou informações indiretas sobre a demanda por atendimento ambulatorial pelas vítimas de causas externas em geral, e agressões e acidentes de transporte em particular, além de informações sobre as despesas estaduais e municipais com o SUS. Segundo nosso resultado, o custo total do atendimento às vítimas de causas externas pelo sistema público de saúde em 2004 teria sido de 2,2 bilhões, o das agressões de 119 milhões e dos acidentes de trânsito de 453 milhões de Reais. Esse conjunto de gastos equivaleria a cerca de $4 \%$ dos gastos totais com a saúde pública naquele ano.

Como recomendação para o aprimoramento qualitativo e quantitativo das pesquisas, não apenas relacionadas ao custo do sistema de saúde com a violência, mas também com relação a uma série de outros temas de relevância para a saúde, sugerimos uma medida relativamente simples na elaboração das informações pertinentes ao SIA, que relaciona-se à necessidade de preencher o CID no prontuário médico de entrada nos hospitais. 


\section{Resumo}

O orçamento público em saúde era da ordem de 53 bilhões de Reais em 2003. Dentro desse orçamento, qual é a parcela referente ao tratamento de vítimas da violência? Devido às limitações dos dados disponíveis não é possível calcular diretamente essa parcela. Neste artigo propomos uma metodologia para estimar o custo da violência para o sistema público de saúde que utiliza informações do Sistema Único de Saúde, dos orçamentos estaduais e municipais e estimativas sobre a demanda por atendimento ambulatorial da Pesquisa Nacional por Amostra de Domicílios. Segundo nossos resultados, em 2004 o tratamento de vítimas de causas externas, de agressões e de acidentes de transporte teria custado ao setor público $R \$ 2,2$ bilhões, $R \$$ 119 milhões e $R \$ 453$ milhões, respectivamente. Esses valores são cerca de quatro vezes maiores que aqueles verificados em trabalhos que computaram apenas os custos com internações.

Sistema Único de Saúde; Sistemas de Informação; Violência

\section{Colaboradores}

Todos os autores contribuíram igualmente na elaboração do trabalho.

\section{Agradecimentos}

Este trabalho é resultado do projeto de pesquisa Análise dos Custos Econômicos e Sociais da Violência no Brasil, financiada com recursos do Ministério da Saúde e do Conselho Nacional de Desenvolvimento Científico e Tecnológico. Os autores agradecem a Alexandre Marinho e Carlos Octavio Ocke Reis pelos comentários e sugestões, bem como às assistentes de pesquisa Vivian Almeida e Michele Guimarães.

\section{Referências}

1. Jorge MHP, Koizumi MS. Gastos governamentais do SUS com internações hospitalares por causas externas: análise no Estado de São Paulo, 2000. Rev Bras Epidemiol 2004; 7:228-38.

2. Fundo Nacional da Saúde, Ministério da Saúde. Gestão financeira do sistema único de saúde: manual básico. Brasília: Ministério da Saúde; 2003.

3. Gawryszewski VP, Koizumi MS, Mello-Jorge MHP. As causas externas no Brasil no ano 2000: comparando a mortalidade e a morbidade. Cad Saúde Pública 2004; 20:995-1003.

4. Pinheiro P. A violência do Rio às portas da emergência. Cad Saúde Pública 1994: 10 Suppl 1:S223-5.

5. Feijó MCC, Portela MC. Variação no custo de internações hospitalares por lesões: os casos dos traumatismos cranianos e acidentes por armas de fogo. Cad Saúde Pública 2001; 17:627-37.

6. Mendonça RNS, Alves JGB, Cabral Filho JE. Gastos hospitalares com crianças e adolescentes vítimas de violência no Estado de Pernambuco, Brasil, em 1999. Cad Saúde Pública 2002; 18:1577-81.

7. Iunes RF. Impacto econômico das causas externas no Brasil: um esforço e mensuração. Rev Saúde Pública 1997; 31(4 Suppl):38-46.

8. Deslandes SF, Silva CMFP, Ugá MAD. O custo do atendimento emergencial às vítimas de violências em dois hospitais do Rio de Janeiro. Cad Saúde Pública 1998; 14:287-99.

9. Martins CBG, Andrade SM. Causas externas entre menores de 15 anos em cidade do Sul do Brasil: atendimentos em pronto-socorro, internações e óbitos. Rev Bras Epidemiol 2005; 8:194-204.

10. Carneiro LP, Phebo L, organizadores. Magnitude, custos econômicos e políticas de controle da violência no Rio de Janeiro. Washington DC: Banco Interamericano de Desenvolvimento/Rio de Janeiro: Instituto de Estudos da Religião; 1998. (Série Documentos de Trabalho, R-347).

11. Levcovitz E, Lima LD, Machado CV. Política de saúde nos anos 90: relações intergovernamentais e o papel das normas operacionais básicas. Ciênc Saúde Coletiva 2001; 6:269-91.

12. Teixeira HV, Teixeira MG. Financiamento da saúde pública no Brasil: a experiência do Siops. Ciênc Saúde Coletiva 2003; 8:379-91.

13. Ribeiro JA, Piola SF, Servo LM. As novas configurações de antigos problemas: financiamento e gasto com ações e serviços públicos de saúde no Brasil. Divulg Saúde Debate 2007; 37:21-43.

Recebido em 23/Out/2007

Versão final reapresentada em 09/Jun/2008

Aprovado em 01/Jul/2008 